\title{
Effect of Ethanolic Extract of Centella asiatica against Streptozocin and High Fat Diet Induced Diabetes and Hyperlipidemia in Rats
}

\section{Mohd Ayyub $^{1 *}$, Mohammad Mukim ${ }^{3,4}$, Mohsina F Patwekar ${ }^{2}$, Faheem I Patwekar ${ }^{2}$, Pratishtha Sharma ${ }^{5}$, Rajiv Kukkar ${ }^{5}$, Rakesh Patel ${ }^{4}$ and Tarique Mahmood ${ }^{1}$}

${ }^{1}$ Faculty of Pharmacy, Integral University, Lucknow, UP, India

${ }^{2}$ Luqman College of Pharmacy, Gulbarga, India

${ }^{3}$ Kota College of Pharmacy, Kota, Rajasthan, India

${ }^{4}$ Dr. A.P.J Abdul Kalam University, Indore, MP, India

${ }^{5}$ School of Pharmacy, Raffles University, Alwar, Rajasthan, India

*Corresponding Author: Mohd Ayyub, Faculty of Pharmacy, Integral University, Lucknow, UP, India.
Received: January 27, 2021

Published: August 28, 2021

(C) All rights are reserved by Mohd Ayyub., et al.

\begin{abstract}
Centella asiatica is a well-established plant in both of the traditional system of medicine in Ayurveda and Unani. In Ayurveda, the plant is reported in the treatment of hyperglycemia and as a blood purifier. The plant is having in vitro antioxidant and free radical scavenging activity, hypoglycemic activity, and cardioprotective effect. Centella asiatica is the chief ingredient in many of the polyherbal formulations used in ayurveda and unani viz. Abana. Centella asiatica has been proved as a good hypoglycemic. The present study was conducted on a 14 day model in which the effect of Centella asiatica extract was observed on STZ induced diabetic rats that were fed with high fat diet (HFD). Diabetes induced by STZ ( $50 \mathrm{mg} / \mathrm{kg}$ ) i.p. injection. All the animals were allocated into 7 different groups following two types of dietary regimen. Normal pellet diet (NPD) and high fat diet (HFD) to observe the effects of STZ in presence of each groups except NC. Daily food and water was measured. Hyperlipidemia induced by feeding with HFD to all animal except NC. The parameters for evaluation of antihyperlipidemic and antihyperglycemic activities are the physical parameters and the biochemical estimation. These are blood glucose level, food efficiency ratio, liver weight, heart weight, lipid profile, biochemical marker and histopathological studies of aorta. The diabetic hyperlipidemic group showed marked elevation and the pre-treatment groups showed significant decrease in theses parameters as compared to D-HFD group ( $<0.01)$. In biochemical estimations various enzymes like Total cholesterol (TC), Triglyceride (TG). The diabetic hyperlipidemic group shows marked elevation and the pre-treatment groups show significant control in theses parameters as compared to D-HFD group $(p<0.01)$. The result of this study showed that alcoholic extract Centella asiatica $(500 \mathrm{mg} / \mathrm{kg}$ ) has significant antidiabetic and anti hyperlipidemic activity against streptozocin and high fat diet induced diabetic hyperlipidemic rats. All these results were compared and found to be similar with two standard drugs metformin $(500 \mathrm{mg} / \mathrm{kg})$ and atorvastatin $(10 \mathrm{mg} / \mathrm{Kg})$.
\end{abstract}

Keywords: Hyperlipidemia; Centella asiatica; Ayurveda

\section{Introduction}

Compared the our past lifestyle now a day's completely Changes in lifestyles reduced physical activity, increased obesity, and rapid changes in environmental complications contribute to the increasing the number of eventuality of diabetes mellitus [1]. Globally, an estimated 422 million adults were living with diabetes in 2014, compared to 108 million in 1980 , rising from 4.7 to $8.5 \%$ in the adult population. WHO projects that diabetes will be the seventh leading cause of death in 2030 [2].

Diabetes mellitus is a group of metabolic diseases characterized by hyperglycemia resulting from defects in insulin secretion, insulin action, or both. The chronic hyperglycemia of diabetes is associated with long-term damage, dysfunction, and failure of various organs, especially the eyes, kidneys, nerves, heart, and blood 
vessels. Several pathogenic processes are involved in the development of diabetes. These range from autoimmune destruction of the $\beta$-cells of the pancreas with consequent insulin deficiency to abnormalities that result in resistance to insulin action. The basis of the abnormalities in carbohydrate, fat, and protein metabolism in diabetes is deficient action of insulin on target tissues. Deficient insulin action results from inadequate insulin secretion and/or diminished tissue responses to insulin at one or more points in the complex pathways of hormone action. Impairment of insulin secretion and defects in insulin action frequently coexist in the same patient, and it is often unclear which abnormality, if either alone, is the primary cause of the hyperglycemia [3]. The classic symptoms of untreated diabetes are loss of weight, polyuria (frequent urination), polydipsia (increased thirst) and polyphagia (increased hunger). Symptoms may develop rapidly (weeks or months) in type 1 diabetes, while they usually develop much more slowly and may be subtle or absent in type 2 diabetes [4].

The two types of diabetes are referred to as:

- $\quad$ Type 1 (insulin dependent).

- $\quad$ Type 2 (non-insulin dependent).

\section{Type 2 diabetes mellitus}

It is a chronic metabolic disease that affects the body's ability to turn food into energy. Normally, after eating, sugar in the form of glucose from the food enters the bloodstream. The hormone Insulin, released from the pancreas in response to the rising levels of glucose in the bloodstream, is responsible for transporting the glucose into the body's cells, to be used as fuel. Other hormones produced by the gastric system such as GLP-1 also play a part in controlling glucose metabolic processes. In Type 2 diabetes, the body does not respond well to insulin. As a result, the cells cannot absorb the glucose properly and it remains and builds up in the bloodstream and damages the inner lining of the small blood vessels. Uncontrolled diabetes may lead to blindness, limb amputation, kidney failure, and nerve damage. Diabetes is also a prominent factor in accelerating the hardening and narrowing of the arteries (atherosclerosis), leading to stroke, heart disease, and other small blood vessel disorders [5].

\section{Diabetic hyperlipidemia}

Hyperlipidemia, elevated levels of lipids in the blood plasma, is a condition commonly observed in patients with type 2 diabetes. It is also characteristic of metabolic syndrome disorders. When hyperlipidemia occurs, chronic inflammation inside the patient takes place as levels of HDL cholesterol decrease and levels of LDL cholesterol increase. The end result of hyperlipidemia may be cardiovascular disease, and in many cases, heart-related fatality. Type 2 diabetics are most likely to become ill, disabled, or even suffer fatality due to cardiovascular complications, and for many patients, they are also dealing with the condition of hyperlipidemia, though very few are aware of it. As is commonly the case with type 2 diabetics, obesity remains a significant concern, and for those also suffering from hyperlipidemia, achieving weight loss becomes an even stronger necessity for the individual. Obese patients with diabetic hyperlipidemia are at a great risk for health complications from hypertension, health function, heart disease, and the risk of mortality, according to Quality of Life Research (1998) [6,7].

\section{Centella asiatica}

Centella asiatica consists of dried whole plant of Centella asiatica (Linn.)Urban. Syn. Hydrocotyleasiatica Linn from the Family of Apiaceae [8].

\section{Chemical constituents}

It contains several active constituents, of which the most important are the triterpenoid saponins, including asiaticoside, centelloside, madecassoside, and asiatic acid. In addition, Centella contains other components, including volatile oils, flavonoids, tannins, phytosterols, amino acids, and sugars [9].

\section{Aim of the Study}

Effect of ethanolic extract of Centella asiatica against streptozocin and high fat diet induced diabetes and hyperlipidemia in rats.

\section{Materials and Methods}

Malesprague-dowley rats (150 - 200g) were used for the study. They were housed five each in sanitized polypropylene cages containing paddy husk as bedding under standard laboratory conditions at room temperature $\left(23^{\circ} \mathrm{C} \pm 2{ }^{\circ} \mathrm{c}\right)$ with $12 \mathrm{hrs}$ light/dark cycle. The animals was randomized into experimental and control groups. They have free access to standard pellets as basal diet and water ad libitum.

\section{Approval from animal ethical committee}

Ethical clearance was obtained from institutional animal ethical committee (IAEC), Approval No. IU/Pharm/ M.Pharm/IAEC/14/08 dated $05^{\text {th }}$ July, 2014, Faculty of pharmacy Integral University, Dasauli, P.O. Bas-ha Kursi Road; Lucknow - 226026 (U.P).

\section{Collection and authentication of crude drug (Centella asiatica)}

Dried leaves of Centella asiatica was purchased from the local market of Lucknow. The drug was authenticated by a taxonomist of 
national botanical research institute Lucknow. The voucher specimen no. is NBRI-SOP-202 and Ref. No NBRI/CIF/409/2013, dated 23.12.2013. The sample specimen has been preserved in faculty of pharmacy, integral university, Lucknow for future references.

\section{Preparation of ethanolic extract of Centella asiatica}

The whole plant was dried in laboratory at room temperature and powdered in a mixer grinder. To prepare the extract $25 \mathrm{~g}$ of the whole plant powder in $25 \mathrm{ml}$ of ethyl alcohol was stirred magnetically for $4 \mathrm{~h}$ at room temperature. The residue was removed by filtration and it was once again extracted as above and filtered. The combined filtered was evaporated to dryness at $40-50^{\circ} \mathrm{C}$ under reduced pressure in a rotary evaporator (yield of ethyl alcohol extract was approximately 10\%) [12].

\section{Streptozocin solution}

Standard streptozocin was dissolved in $0.2 \mathrm{M}$ citrate buffer and freshly prepared solution was given to the animals according to dose for induction of diabetes in rats.

\section{Atorvastatin solution}

Standard atorvastatin was dissolved in $0.9 \%$ normal saline and freshly prepared solution was used as a standard drug.

\section{Metformin solution}

Metformin tablets were powdered and dissolved in $0.9 \%$ normal saline and freshly prepared solution was given to the animals according to dose.

Formaldehyde solution

Freshly prepared $10 \%$ formaldehyde solution.

\section{Plant extract solution}

The dried plant extract was dissolved in $0.9 \%$ normal saline and freshly prepared solution was given to animals according to dose.

\section{Experimental protocol}

\section{Induction of diabetes mellitus}

Rats were fasted $24 \mathrm{hr}$ before the induction of diabetes by streptozocin injection. The STZ was freshly dissolved in normal saline $0.2 \mathrm{M}$ sodium citrate buffer, pH4.5 with a single dose of STZ $50 \mathrm{mg} /$ $\mathrm{kg}$ immediate injection in overnight fasted SD rats. After $48 \mathrm{hr}$ of STZ injection diabetes mellitus was confirmed by blood sample col- lected from the tip of the tail using a blood glucometer. The rats with a fasting blood glucose level $250 \mathrm{mg} / \mathrm{dL}$ were considered diabetic and were used in experiment [13].

\section{Experimental design}

The normal group (NC) served as non diabetic control rats treated with saline instead of STZ. All diabetic rats was allocated in to two dietary regimen i.e. one group was received normal pallet diet (NPD) and all other groups fed with composition of high fat diet was according to table $[13,14]$.

\begin{tabular}{|c|c|c|}
\hline S. No & Ingredients & Quantity (g/100g) \\
\hline 1 & Corn flour & 25 \\
\hline 2 & Milk Powder & 15 \\
\hline 3 & Sucrose & 15 \\
\hline 4 & Caesein & 05 \\
\hline 5 & Egg Yolk & 03 \\
\hline 6 & Cholesterol & 01 \\
\hline 7 & Salt Mixture & 01 \\
\hline 8 & Lard & 35 \\
\hline
\end{tabular}

Table 1: Composition of High fat Diet.

\section{Experimental procedure}

At the beginning of study ( 0 day) the body weight and fasting blood glucose was measured of all animal of different groups. The animal NC and D-NPD were fed with NPD and all others were fed with HFD along with NPD. Food and water intake were monitored daily at the same time during the experimental period. The food efficiency ratio (FER) was calculated as the total body weight gain (gm)/total food intake amount (gm) during the experimental period at the end of study (15 day). Final body weight of all animals were taken and then animal have been sacrificed with high dose of diethyl ether (over night fasted). Blood sample was collected from retro orbital plexus/ cardiac puncher and was allowed to clot for 30 minute at room temperature. The serum was separated by centrifugation at $3000 \mathrm{rpm}$ at $30^{\circ} \mathrm{C}$ for 15 minute and was used for estimations. The animal has been dissected and aorta has been isolated and kept in formaline (10\%) for histopathological study. The liver and heart were excised immediately and rinsed with ice cold normal saline and weighed $[13,14,16]$.

All diabetic rats was divided in 5 groups as follows. 


\begin{tabular}{|c|c|c|c|}
\hline $\begin{array}{l}\text { S. } \\
\text { No. }\end{array}$ & Groups & No. of Animals & Treatment will be given \\
\hline 1. & Normal Control & 5 & Administered with Normal saline instead of STZ and fed with NPD \\
\hline 2. & $\begin{array}{l}\text { Diabetic control } \\
\text { (D-NPD) }\end{array}$ & 5 & $\begin{array}{l}\text { Rats were administered with single dose of STZ ( } 50 \mathrm{mg} / \mathrm{kg} \text { i.p.) and } \\
\text { fed with normal pallet diet throughout the study period (15 days) }\end{array}$ \\
\hline 3. & $\begin{array}{l}\text { Diabetic- high fat diet } \\
\text { (D-HFD) }\end{array}$ & 5 & $\begin{array}{c}\text { Rats were treated with single of STZ ( } 50 \mathrm{mg} / \mathrm{kg} \text { i.p) and fed with } \\
\text { high fat diet along with NPD throughout the study period (15 } \\
\text { days) }\end{array}$ \\
\hline 4. & $\begin{array}{l}\text { Casiatica }[\mathrm{TE} 1] \\
(250 \mathrm{mg} / \text { day })\end{array}$ & 5 & $\begin{array}{c}\text { Rats were treated with single of STZ }(50 \mathrm{mg} / \mathrm{kg} \text { i.p) and pretreated } \\
\text { with extract of } C \text { asiatica }(250 \mathrm{mg} / \mathrm{kg} / \mathrm{d}) \text { throughout the study } \\
\text { period ( } 15 \text { days })\end{array}$ \\
\hline 5. & $\begin{array}{l}\text { Casiatica }[\mathrm{TE} 2] \\
{[500 \mathrm{mg} / \mathrm{kg}]}\end{array}$ & 5 & $\begin{array}{l}\text { Rats were treated with single of STZ ( } 50 \mathrm{mg} / \mathrm{kg} \text { i.p) and pretreated } \\
\text { with extract of } C \text { asiatica }(500 \mathrm{mg} / \mathrm{kg} / \mathrm{d}) \text { throughout the study } \\
\text { period ( } 15 \text { days) }\end{array}$ \\
\hline 6. & $\begin{array}{l}\text { Metformin } \\
{[0.5 \mathrm{mg} / \mathrm{kg}]}\end{array}$ & 5 & $\begin{array}{l}\text { Rats were treated with single of STZ ( } 50 \mathrm{mg} / \mathrm{kg} \text { i.p) and pretreated } \\
\text { with Metformin }(0.5 \mathrm{mg} / \mathrm{kg} / \mathrm{d}) \text { throughout the study period (15 } \\
\text { days })\end{array}$ \\
\hline 7. & $\begin{array}{l}\text { Atorvastatin treated } \\
\qquad[10 \mathrm{mg} / \mathrm{kg}]\end{array}$ & 5 & $\begin{array}{c}\text { Rats were treated with single of STZ (50mg/kg i.p) and pretreated } \\
\text { with Atorvastatin }(10 \mathrm{mg} / \mathrm{kg} / \mathrm{d}) \text { throughout the study period (15 } \\
\text { days })\end{array}$ \\
\hline
\end{tabular}

Table 2: Experimental Protocol [16].

Results Blood glucose level (At 0 day)

\begin{tabular}{|c|c|c|c|c|c|c|c|}
\hline S.no. & NC & D-NPD & D-HFD & $\begin{array}{l}\text { Atorvastatin } \\
10 \mathrm{mg} / \mathrm{kg}\end{array}$ & $\begin{array}{l}\text { Metformin } \\
500 \mathrm{mg} / \mathrm{kg}\end{array}$ & $\begin{array}{l}\text { C. asiatica } \\
250 \mathrm{mg} / \mathrm{kg}\end{array}$ & $\begin{array}{l}\text { C. asiatica } \\
500 \mathrm{mg} / \mathrm{kg}\end{array}$ \\
\hline 1 & 77 & 98 & 89 & 94 & 66 & 95 & 100 \\
\hline 2 & 94 & 102 & 104 & 89 & 102 & 85 & 75 \\
\hline 3 & 87 & 79 & 96 & 69 & 75 & 100 & 101 \\
\hline 4 & 82 & 87 & 92 & 72 & 67 & 80 & 77 \\
\hline 5 & 105 & 109 & 101 & 101 & 89 & 80 & 84 \\
\hline Mean & 89 & 95 & 96.4 & 85.0 & 79.8 & 88.0 & 87.4 \\
\hline \pm SEM & \pm 4.88 & \pm 5.35 & \pm 2.76 & \pm 6.23 & \pm 6.90 & \pm 4.06 & \pm 5.55 \\
\hline
\end{tabular}

Table 3: Blood glucose level (At 0 day).

All values are expressed as mean \pm SEM calculated by one way ANOVA $(n=5)$. 
Blood glucose level (After $48 \mathrm{hrs}$ )

\begin{tabular}{|l|c|c|c|c|c|c|c|}
\hline S. No & NC & D-NPD & D-HFD & $\begin{array}{c}\text { Atorvastatin } \\
\mathbf{1 0} \mathbf{m g} / \mathbf{k g}\end{array}$ & $\begin{array}{c}\text { Metformin } \\
\mathbf{5 0 0} \mathbf{m g} / \mathbf{k g}\end{array}$ & $\begin{array}{c}\text { C. asiatica } \\
\mathbf{2 5 0} \mathbf{m g} / \mathbf{k g}\end{array}$ & $\begin{array}{c}\text { C. asiatica } \\
\mathbf{5 0 0} \mathbf{m g} / \mathbf{k g}\end{array}$ \\
\hline 1 & 75 & 155 & 175 & 165 & 225 & 185 & 180 \\
\hline 2 & 99 & 159 & 220 & 175 & 215 & 175 & 170 \\
\hline 3 & 85 & 170 & 228 & 188 & 218 & 179 & 170 \\
\hline 4 & 78 & 157 & 215 & 168 & 190 & 181 & 179 \\
\hline 5 & 101 & 180 & 225 & 177 & 207 & 178 & 171.8 \\
\hline Mean & 87.6 & 164 & 221 & 174 & \pm 7.61 & \pm 2.56 & \pm 2.59 \\
\hline SEM & \pm 5.32 & \pm 4.72 & \pm 9.65 & \pm 4.00 & & & \\
\hline
\end{tabular}

Table 4: Blood glucose level (After $48 \mathrm{hrs}$ ).

All values are expressed as mean \pm SEM calculated by one way ANOVA $(n=5)$.

Final blood glucose level (At 14th day)

\begin{tabular}{|l|c|c|c|c|c|c|c|}
\hline S. No & NC & D-NPD & D-HFD & $\begin{array}{c}\text { Atorvastatin } \\
\mathbf{1 0} \mathbf{m g} / \mathbf{k g}\end{array}$ & $\begin{array}{c}\text { Metformin } \\
\mathbf{5 0 0} \mathbf{m g} / \mathbf{k g}\end{array}$ & $\begin{array}{c}\text { C. asiatica } \\
\mathbf{2 5 0} \mathbf{m g} / \mathbf{k g}\end{array}$ & $\begin{array}{c}\text { C. asiatica } \\
\mathbf{5 0 0} \mathbf{m g} / \mathbf{k g}\end{array}$ \\
\hline 1 & 77 & 170 & 190 & 168 & 110 & 135 & 129 \\
\hline 2 & 102 & 250 & 270 & 174 & 107 & 128 & 122 \\
\hline 3 & 88 & 240 & 288 & 165 & 101 & 124 & 120 \\
\hline 4 & 75 & 235 & 290 & 190 & 99 & 130 & 125 \\
\hline 5 & 103 & 285 & 275 & 185 & 102 & $* 126$ & $* 130.2$ \\
\hline Mean & 89.0 & 236 & $* 262.6$ & $* 176.4$ & $* 103.8$ & \pm 124.4 \\
\hline SEM & \pm 5.94 & \pm 18.67 & \pm 18.54 & \pm 4.82 & \pm 2.03 & \pm 2.01 & \pm 1.56 \\
\hline
\end{tabular}

Table 5: Final blood glucose level (At $14^{\text {th }}$ day).

All values are expressed as mean \pm SEM calculated by one way ANOVA followed by Tukey’s test $(n=5)$. * $=p<0.01$ when D- NPD compared with NC, and all pre-treatment groups compared with D-HFD.

Initial body weight (gm)

\begin{tabular}{|c|c|c|c|c|c|c|c|}
\hline S. No & NC & D- NPD & D-HFD & $\begin{array}{c}\text { Atorvasatin } \\
10 \mathrm{mg} / \mathrm{kg}\end{array}$ & $\begin{array}{l}\text { Metformin } \\
500 \mathrm{mg} / \mathrm{kg}\end{array}$ & $\begin{array}{l}\text { C. asiatica } \\
250 \mathrm{mg} / \mathrm{kg}\end{array}$ & $\begin{array}{l}\text { C. asiatica } \\
500 \mathrm{mg} / \mathrm{kg}\end{array}$ \\
\hline 1 & 209 & 198 & 248 & 172 & 200 & 175 & 250 \\
\hline 3 & 210 & 196 & 250 & 174 & 200.5 & 170 & 245 \\
\hline 4 & 211 & 197 & 252 & 175 & 200 & 180 & 250 \\
\hline 5 & 211 & 200 & 249 & 175 & 200 & 175 & 248 \\
\hline \pm SEM & \pm 0.374 & \pm 0.86 & \pm 0.67 & \pm 0.62 & \pm 0.10 & \pm 1.58 & \pm 1.88 \\
\hline
\end{tabular}

Table 6: Initial body weight.

All values are expressed as mean \pm SEM calculated by one way ANOVA $(n=5)$. 
Final body weight (gm)

\begin{tabular}{|c|c|c|c|c|c|c|c|}
\hline S. No & NC & D- NPD & D-HFD & $\begin{array}{c}\text { Atorvasatin } \\
10 \mathrm{mg} / \mathrm{kg}\end{array}$ & $\begin{array}{l}\text { Metformin } \\
500 \mathrm{mg} / \mathrm{kg}\end{array}$ & $\begin{array}{c}\text { C. asiatica } \\
250 \mathrm{mg} / \mathrm{kg}\end{array}$ & $\begin{array}{l}\text { C. asiatica } \\
500 \mathrm{mg} / \mathrm{kg}\end{array}$ \\
\hline 1 & 225 & 170 & 280 & 184 & 212 & 170 & 238 \\
\hline 2 & 220 & 175 & 275 & 185 & 215 & 170 & 230 \\
\hline 3 & 228 & 184 & 265 & 182 & 220 & 165 & 235 \\
\hline 4 & 226 & 185 & 278 & 187 & 218 & 172 & 239 \\
\hline 5 & 222 & 180 & 267 & 186 & 210 & 175 & 241 \\
\hline Mean & 224.2 & $* 178$ & $* 273$ & *184.8 & $* 215$ & $* 170.4$ & $* 236.6$ \\
\hline \pm SEM & \pm 1.42 & \pm 2.81 & \pm 2.98 & \pm 0.86 & \pm 1.84 & \pm 1.63 & \pm 1.91 \\
\hline
\end{tabular}

Table 7: Final body weights.

All values are expressed as mean \pm SEM calculated by one way ANOVA followed by Tukey's t-test $(n=5) .{ }^{*}=p<0.01$ when $D-N P D$ compared with NC, D-HFD compared with D-NPD and all pre-treatment groups compared with D-HFD.

Total food intake (gm)

\begin{tabular}{|c|c|c|c|c|c|c|c|}
\hline S. No & NC & D-NPD & D-HFD & $\begin{array}{c}\text { Atorvastatin } \\
10 \mathrm{mg} / \mathrm{kg}\end{array}$ & $\begin{array}{c}\text { Metformin } \\
500 \mathrm{mg} / \mathrm{kg}\end{array}$ & $\begin{array}{l}\text { C. asiatica } \\
200 \mathrm{mg} / \mathrm{kg}\end{array}$ & $\begin{array}{l}\text { C. asiatica } \\
500 \mathrm{mg} / \mathrm{kg}\end{array}$ \\
\hline 1 & 28 & 53 & 75 & 77 & 33 & 57 & 52 \\
\hline 2 & 24 & 55 & 81 & 85 & 39 & 59 & 55 \\
\hline 3 & 27 & 59 & 77 & 79 & 32 & 65 & 60 \\
\hline 4 & 22 & 53 & 71 & 76 & 39 & 55 & 50 \\
\hline 5 & 25 & 51 & 83 & 85 & 31 & 56 & 51 \\
\hline Mean & 25.2 & 54.2 & 77 & 80.4 & 34.8 & 58.4 & 53.6 \\
\hline \pm SEM & \pm 1.06 & \pm 1.35 & \pm 2.13 & \pm 1.93 & \pm 1.74 & \pm 1.77 & \pm 1.80 \\
\hline
\end{tabular}

Table 8: Total food intake.

All values are expressed as mean \pm SEM calculated by one way ANOVA $(n=5)$.

Total water intake $(\mathrm{ml})$

\begin{tabular}{|l|c|c|c|c|c|c|c|}
\hline S. No & NC & D-NPD & D-HFD & $\begin{array}{c}\text { Atorvastatin } \\
\mathbf{1 0} \mathbf{m g} / \mathbf{k g}\end{array}$ & $\begin{array}{c}\text { Metformin } \\
\mathbf{5 0 0} \mathbf{m g} / \mathbf{k g}\end{array}$ & $\begin{array}{c}\text { C. asiatica } \\
\mathbf{2 5 0} \mathbf{m g} / \mathbf{k g}\end{array}$ & $\begin{array}{c}\text { C. asiatica } \\
\mathbf{5 0 0} \mathbf{m g} / \mathbf{k g}\end{array}$ \\
\hline 1 & 32 & 190 & 244 & 202 & 170 & 195 & 175 \\
\hline 2 & 25 & 185 & 250 & 200 & 175 & 190 & 185 \\
\hline 3 & 29 & 199 & 228 & 196 & 165 & 180 & 170 \\
\hline 4 & 34 & 201 & 225 & 186 & 158 & 172 & 161 \\
\hline 5 & 27 & 189 & 230 & 178 & 169 & 176 & 166 \\
\hline Mean & 29.4 & 192.8 & 235.4 & 192.4 & 167.4 & 182.6 & 171.4 \\
\hline \pm SEM & \pm 1.63 & \pm 3.07 & \pm 4.89 & \pm 4.53 & \pm 2.8 & \pm 4.30 & \pm 4.10 \\
\hline
\end{tabular}

Table 9: Total water intake.

All values are expressed as mean \pm SEM calculated by one way ANOVA $(n=5)$. 
Food efficiency ratio

\begin{tabular}{|c|c|c|c|c|c|c|c|}
\hline S. No & NC & D-NPD & D-HFD & $\begin{array}{c}\text { Atorvastatin } \\
10 \mathrm{mg} / \mathrm{kg} \\
\end{array}$ & $\begin{array}{l}\text { Metformin } \\
500 \mathrm{mg} / \mathrm{kg}\end{array}$ & $\begin{array}{c}\text { C. asiatica } \\
(250 \mathrm{mg} / \mathrm{kg})\end{array}$ & $\begin{array}{c}\text { C. asiatica } \\
\text { (500mg/kg) }\end{array}$ \\
\hline 1 & 0.57 & -0.71 & 0.42 & 0.15 & 0.36 & -0.08 & -0.23 \\
\hline 2 & 0.46 & -0.36 & 0.31 & 0.11 & 0.38 & -0.08 & -0.18 \\
\hline 3 & 0.66 & -0.20 & 0.19 & 0.10 & 0.61 & -0.07 & -0.16 \\
\hline 4 & 0.68 & -0.22 & 0.36 & 0.15 & 0.46 & -0.14 & -0.22 \\
\hline 5 & 0.44 & 0.39 & 0.21 & 0.13 & 0.32 & -0.10 & -0.13 \\
\hline Mean & 0.562 & $\#_{-0.22}$ & 0.298 & ${ }^{\#} 0.128$ & ${ }^{\#} 0.42$ & $*_{-} 0.09$ & ${ }^{\#}-0.18$ \\
\hline \pm SEM & \pm 0.049 & \pm 0.17 & \pm 0.043 & \pm 0.010 & \pm 0.05 & \pm 0.012 & \pm 0.018 \\
\hline
\end{tabular}

Table 10: Food efficiency ratio.

All values are expressed as mean \pm SEM calculated by one way ANOVA followed by Tukey's t-test $(n=5) .{ }^{*}=p<0.01$ when C.A $(250 \mathrm{mg} /$ kg) compared with D-HFD compared. ${ }^{*}=\mathrm{p}<0.05$ when C.A $(500 \mathrm{mg} / \mathrm{kg})$ Ator, Met, D-NPD compared with D-HFD.

Liver weight (gm)

\begin{tabular}{|l|c|c|c|c|c|c|c|}
\hline S. No & NC & D-NPD & D-HFD & $\begin{array}{c}\text { Atorvastatin } \\
\mathbf{1 0} \mathbf{m g} / \mathbf{k g}\end{array}$ & $\begin{array}{c}\text { Metformin } \\
\mathbf{5 0 0} \mathbf{m g} / \mathbf{k g}\end{array}$ & $\begin{array}{c}\text { C. asiatica } \\
\mathbf{2 5 0} \mathbf{m g} / \mathbf{k g}\end{array}$ & $\begin{array}{c}\text { C. asiatica } \\
\mathbf{5 0 0} \mathbf{m g} / \mathbf{k g}\end{array}$ \\
\hline $\mathbf{1}$ & 9.12 & 10.27 & 14.72 & 12.21 & 13.81 & 14.32 & 13.90 \\
\hline 2 & 9.52 & 10.89 & 14.15 & 12.01 & 13.96 & 14.05 & 13.90 \\
\hline 3 & 9.33 & 10.50 & 14.19 & 12.09 & 13.76 & 14.10 & 13.85 \\
\hline 4 & 9.01 & 10.70 & 14.50 & 12.34 & 13.85 & 14.01 & 13.90 \\
\hline 5 & 9.07 & 10.20 & 14.65 & 12.07 & 13.59 & 14.20 & 13.80 \\
\hline Mean & 9.21 & $* 10.5$ & $* 14.4$ & $* 12.1$ & $* 13.8$ & $* 14.14$ & $* 13.87$ \\
\hline \pm SEM & \pm 0.09 & \pm 0.12 & \pm 0.11 & \pm 0.05 & \pm 0.06 & \pm 0.05 & \pm 0.02 \\
\hline
\end{tabular}

Table 11: Liver weight.

All values are expressed as mean \pm SEM calculated by one way ANOVA followed by Tukey's t-test $(n=5)$. * $=p<0.01$ when D- NPD compared with NC, D-HFD compared with D-NPD and all pre-treatment groups compared with D-HFD.

Heart weight (gm)

\begin{tabular}{|c|c|c|c|c|c|c|c|}
\hline S. No & NC & D-NPD & D-HFD & $\begin{array}{c}\text { Atorvastatin } \\
10 \mathrm{mg} / \mathrm{kg}\end{array}$ & $\begin{array}{l}\text { Metformin } \\
500 \mathrm{mg} / \mathrm{kg}\end{array}$ & $\begin{array}{l}\text { C. asiatica } \\
250 \mathrm{mg} / \mathrm{kg}\end{array}$ & $\begin{array}{l}\text { C. asiatica } \\
500 \mathrm{mg} / \mathrm{kg}\end{array}$ \\
\hline 1 & 0.88 & 0.92 & 1.12 & 0.89 & 0.94 & 1.09 & 0.96 \\
\hline 2 & 0.78 & 0.79 & 0.99 & 0.88 & 0.91 & 0.98 & 0.95 \\
\hline 3 & 0.69 & 0.72 & 0.97 & 0.79 & 0.93 & 0.97 & 0.94 \\
\hline 4 & 0.79 & 0.83 & 1.00 & 0.83 & 0.95 & 0.99 & 0.96 \\
\hline 5 & 0.70 & 0.74 & 0.98 & 0.77 & 0.90 & 0.97 & 0.93 \\
\hline Mean & 0.76 & $* 0.80$ & $* 1.01$ & $* 0.83$ & $* 0.92$ & $* 1.00 *$ & $* 0.94$ \\
\hline \pm SEM & \pm 0.03 & \pm 0.03 & \pm 0.02 & \pm 0.02 & \pm 0.01 & \pm 0.0 .02 & \pm 0.01 \\
\hline
\end{tabular}

Table 12: Heart weight.

All values are expressed as mean \pm SEM calculated by one way ANOVA followed by Tukey's t-test $(n=5) .^{*}=p<0.01$ when D- NPD compared with NC, D-HFD compared with D-NPD and all pre-treatment groups compared with D-HFD. 
Lipid profile

Total cholesterol (TC) (mg/dl)

\begin{tabular}{|c|c|c|c|c|c|c|c|}
\hline S. No & NC & D-NPD & D-HFD & $\begin{array}{c}\text { Atorvastatin } \\
10 \mathrm{mg} / \mathrm{kg}\end{array}$ & $\begin{array}{l}\text { Metformin } \\
500 \mathrm{mg} / \mathrm{kg}\end{array}$ & $\begin{array}{l}\text { C. asiatica } \\
250 \mathrm{mg} / \mathrm{kg}\end{array}$ & $\begin{array}{l}\text { C. asiatica } \\
500 \mathrm{mg} / \mathrm{kg}\end{array}$ \\
\hline 1 & 45.02 & 50.04 & 176.01 & 62.05 & 87.0 & 157.01 & 90.28 \\
\hline 2 & 48.11 & 52.06 & 178.12 & 64.8 & 85.5 & 165.25 & 92.05 \\
\hline 3 & 44.51 & 54.01 & 180.15 & 63.2 & 88.7 & 161.16 & 93.25 \\
\hline 4 & 46.20 & 56.12 & 182.19 & 62.5 & 86.5 & 160.08 & 95.01 \\
\hline 5 & 48.80 & 55.19 & 184.16 & 65.0 & 89.0 & 159.25 & 96.35 \\
\hline Mean & 46.53 & 53.48 & 180.1 & 63.51 & 87.34 & 160.6 & 93.39 \\
\hline \pm SEM & \pm 0.84 & \pm 1.09 & \pm 1.44 & \pm 0.59 & \pm 0.66 & \pm 1.35 & \pm 1.06 \\
\hline
\end{tabular}

Table 13: Total cholesterol.

All values are expressed as mean \pm SEM calculated by one way ANOVA followed by Tukey’s t-test $(n=5)$.

Triglycerides (TG) (mg/dl)

\begin{tabular}{|c|c|c|c|c|c|c|c|}
\hline S. No & NC & D-NPD & D-HFD & $\begin{array}{l}\text { Atorvastatin } \\
\qquad 10 \mathrm{mg} / \mathrm{kg}\end{array}$ & $\begin{array}{l}\text { Metformin } \\
500 \mathrm{mg} / \mathrm{kg}\end{array}$ & $\begin{array}{l}\text { C. asiatica } \\
250 \mathrm{mg} / \mathrm{kg}\end{array}$ & $\begin{array}{l}\text { C. asiatica } \\
500 \mathrm{mg} / \mathrm{kg}\end{array}$ \\
\hline 1 & 27.03 & 38.08 & 168.04 & 45.01 & 81.10 & 150.21 & 89.25 \\
\hline 2 & 26.44 & 42.27 & 170.25 & 48.71 & 84.15 & 152.32 & 91.65 \\
\hline 3 & 30.22 & 39.99 & 172.34 & 46.87 & 82.25 & 151.32 & 90.69 \\
\hline 4 & 32.05 & 40.37 & 175.65 & 48.66 & 82.44 & 155.01 & 90.98 \\
\hline 5 & 28.21 & 42.41 & 172.42 & 45.34 & 83.64 & 152.11 & 92.06 \\
\hline Mean & 28.79 & 40.62 & 171.7 & 46.92 & 82.72 & 152.2 & 90.93 \\
\hline \pm SEM & \pm 1.04 & \pm 0.80 & \pm 1.26 & \pm 0.78 & \pm 0.53 & \pm 0.79 & \pm 0.48 \\
\hline
\end{tabular}

Table 14: Triglycerides.

All values are expressed as mean \pm SEM calculated by one way ANOVA followed by Tukey’s t-test $(n=5)$.

All values are expressed as mean \pm SEM calculated by one way ANOVA followed by Tukey’s t-test $(n=5) .^{*}=p<0.01$ when D- NPD compared with NC, D-HFD compared with D-NPD and all pre-treatment groups compared with D-HFD.

Histopathological studies

Histopathological studies were done from Puri pathology. The detailed report is enclosed as annexure 4. All photographs were taken on 40x. Photomicrograph of aorta of NC rats showed regularly arranged smooth muscle fibres with outer lining of flattened or cuboidal cell. In D-NPD the photomicrograph of aorta showed thickened wall with presence of lymphocytic infiltration and groups of cartilage cells surrounded by rim of fibrous tissue. Two fragments of fatty tissue with lymphocytic infiltration. Photomicrograph of aorta of D-HFD showed flattened cell lining was intact. Outer wall of aorta showed remarkably thick layer of loose fibro connective tissue lining with lymphocytic infiltration and presence of large amount of fatty tissue. In atorvastatin treated group the photomicrograph of aorta showed the wall of aorta was normal in 

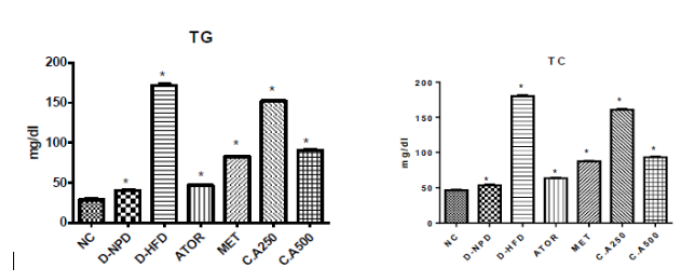

LDL
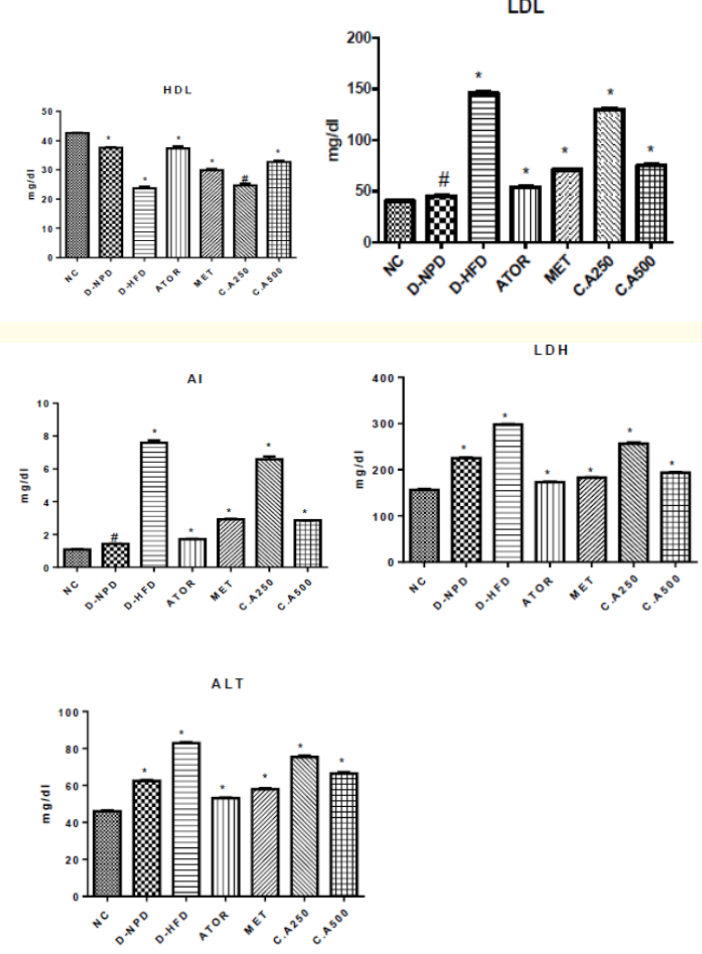

Figure 1

All values are expressed as mean \pm SEM calculated by one way ANOVA followed by Tukey's t-test $(\mathrm{n}=5) .{ }^{*}=\mathrm{p}<0.01$ when D- NPD compared with NC, D-HFD compared with DNPD and all pre-treatment groups compared with D-HFD.

thickness; but one thick fragment of fatty tissue was present. In metformin treated group the photomicrograph of aorta showed the wall of aorta was normal in thickness and cellularity. The cells were regularly arranged with fair no of cell showed vacuolation in their cytoplasm. Remarkably thick layer of fibro connective fatty tissue was present with lymphocytic infiltration. In Centella asi- aticatreated group the photomicrograph of aorta showed wall of aorta was thin with low cellularity. Flattened cell lining on one side was intact. Other side showed prominent loose fibro connective tissue with fat cells lining the wall of aorta.

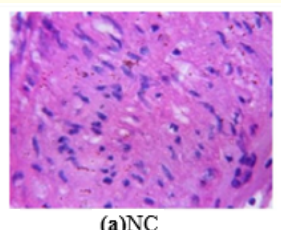

(a) $\mathrm{NC}$

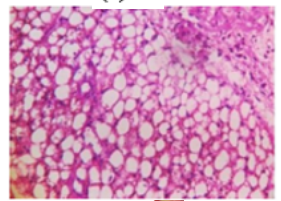

(c)D-HFD

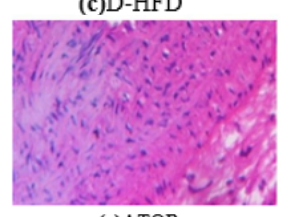

(e)ATOR

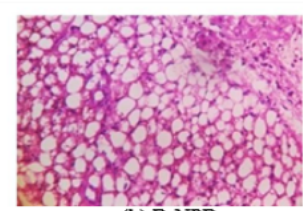

(b) D-NPD

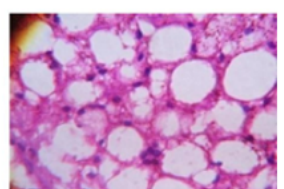

(d) D-HFD \{enlarge\}

(f) MET
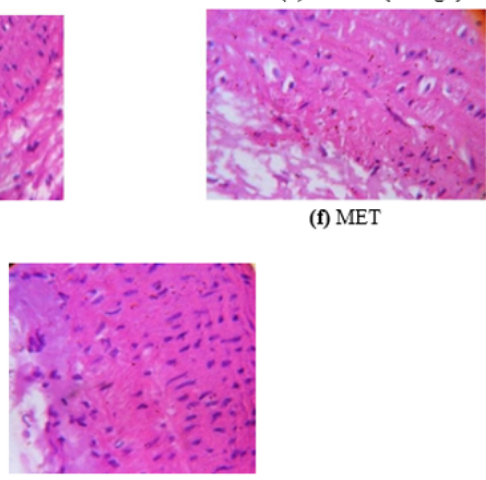

(g) Centellaasiatica

Figure 2: Histopathological studies of aorta of different groups.

\section{Discussion}

Hyperlipidemia is a common problem and leading cause of cardiovascular disease related to development of atherosclerosis. Risk for all forms of CVD, including CHD is increased substantially with type 1 and type 2 diabetes mellitus. Furthermore, the mortality rate in diabetic subjects who have experienced CHD is much higher than in nondiabetic subjects.

India is known for its traditional medicinal systems-Ayurveda, Siddha, and Unani. Medical systems are found mentioned even in the ancient Vedas and other scriptures. Centella asiatica was found that plant is having in vitro antioxidant and free radical scaveng- 
ing activity, hypoglycaemic activity, cardio protective effect. It was also reported the healing effects of Centella extract - Centella asiatica - and asiaticoside on acetic acid induced gastric ulcers in rats. Centella asiatica is the chief ingredient in many of the polyherbal formulations used in ayurveda and unani viz. Abana.

The present study was conducted on a 14 day model in which the effect of Centella asiatica extract was observed on STZ induced diabetic rats that were fed with high fat diet (HFD). Diabetes was induced by $(50 \mathrm{mg} / \mathrm{kg}$ ) i.p. injection of STZ. All the animals were allocated into 7 different groups following two types of dietary regimen. Normal pellet diet (NPD) and High fat diet (HFD) to observe the effects of STZ in presence of each groups except NC. Daily food and water was measured. Hyperlipidemia induced by feeding with HFD to all animal except NC. The pre-treatment of Centella asiatica at two doses $(250 \mathrm{mg} / \mathrm{kg} \& 500 \mathrm{mg} / \mathrm{kg})$ along with the standard drug Atorvastatin $(10 \mathrm{mg} / \mathrm{kg}$ ) and Metformin $(500 \mathrm{mg} / \mathrm{kg}$ ) was given for 14 days.

The protective effect of Centella asiatica was asses by various parameters i.e. blood glucose level, body weight, food efficiency ratio, lipid profile, heart weight, liver weight, atherogenic index and two biochemical parameter i.e. ALT and LDH.

Present study showed that STZ elevates blood glucose level in DNPD but in D-HFD very marked increase due to the presence of potentiating factor i.e. STZ and HFD. Centella asiatica extract $(500 \mathrm{mg} /$ $\mathrm{kg}$ ) showed reduction in blood glucose level as compared to D-HFD $(\mathrm{p}<0.01)$.

The D-NPD showed remarkable loss of weight when compare to NC because of diabetes but D-HFD group showed a drastic increase in weight when compare to NC. Centella asiatica extract (500 mg/ $\mathrm{kg})$ showed very significant reduction in final body weight $(\mathrm{p}<$ 0.01).

The Food efficiency ratio (FER) STZ treated group was found to be significantly improved when compared to D-HFD. Centella asiatica extract ( $500 \mathrm{mg} / \mathrm{kg}$ ) showed very significant improvement in FER as compared to D-HFD ( $\mathrm{p}<0.01)$.

Heart weight \& liver weight are important physical parameters, increase in heart weight is the indication of heart disease and increase in liver weight indicate liver enlargement. Centella asiatica
$(500 \mathrm{mg} / \mathrm{kg})$ reduced the heart weight and liver weight very significantly when compared to D-HFD ( $p<0.01)$.

Estimation of lipid profile i.e. when hyperlipidemia occur the level of TC, TG, and LDL were increased but level of HDL was decrease. Centella asiatica $(500 \mathrm{mg} / \mathrm{kg})$ showed very significant reduction in TG $(\mathrm{p}<0.01)$, TC $(\mathrm{p}<0.01)$, HDL $(\mathrm{p}<0.01)$, and LDL ( $\mathrm{p}$ $<0.01)$ when compared to D-HFD (p<0.01).

Two biochemical enzymes was also assessed i.e. ALT and LDH. Centella asiatica extract $(500 \mathrm{mg} / \mathrm{kg})$ showed very significant when compared to D-HFD ( $\mathrm{p}<0.01)$.

Atherogenic indexes a parameter for development of atherosclerosis. The NC group showed regularly arranged smooth muscle fibres with outer lining of flattened or cuboidal cell and D-NPD, D-HFD groups showed that two fragment of fatty tissue and thick layer of loose fibro connective tissue were found and the pre-treatment groups showed marked decrease in development of atherosclerosis which was clearly indicated by histopathological studies of aorta. Centella asiatica extract ( $500 \mathrm{mg} / \mathrm{kg}$ ) showed very significant effect when compared to D-HFD ( $p<0.01)$.

All these results were also confirmed by histopathological studies of aorta of different groups. The histopathology reveals that the group NC showed regularly arranged smooth muscle fibres with outer lining of flattened or cuboidal cell. The group D-NPD showed two fragments of fatty tissue with lymphocytic infiltration. The group D-HFD showed flattened cell lining was intact and the outer wall with thick layer of loose fibro connective tissue. In atorvastatin treated group showed that aorta wall was normal in thickness but with one thick fragment of fatty tissue. In metformin treated group showed that cells were regularly arranged with fair no of cell showed vacuolation in their cytoplasm. Centella asiatica treated group the photomicrograph of aorta showed the wall of aorta was thin with low cellularity. The flattened cell lining on one side was intact. Other side showed prominent loose fibro connective tissue with fat cell lining the wall of aorta.

In the present study, pre-treatment with ethanolic extract of Centella asiatica significantly prevented STZ and HFD induced hyperlipidemic and hyperglycemic effect. Centella asiatica extract $(500 \mathrm{mg} / \mathrm{kg})$ showed very significant anti hyperlipidemic and anti 
hyperglycemic activity against STZ and HFD induced diabetes and hyperlipidemia. All these results were compared and found to be similar with two standard drugs metformin $(500 \mathrm{mg} / \mathrm{kg})$, and atorvastatin $(10 \mathrm{mg} / \mathrm{kg})$.

\section{Conclusion}

The present study showed that pre-treatment with Ethanolic extract of Centella asiatica significantly controlled the STZ and HFD induced diabetes and hyperlipidemia in SD rats and decreased the levels of diagnostic marker enzymes significantly. The present study concluded with certain observation and result. The observation is that high fat diet along with STZ induced diabetes is a very suitable, potent short term model for evaluating the hypoglycaemic and hypolipidemic effect in rats. The parameters for evaluation of antihyperlipidemic and antihyperglycemic activity the physical parameters, lipid profile and biochemical parameters are the estimations. The result of this study clearly showed significant reduction in physical parameters (final body weight, heart weight and liver weight) and lipid profile i.e. total cholesterol (mg/dl), triglycerides, LDL-C (mg/dl), HDL-C (mg/dl), atherogenic index, and the two biochemical marker i.e. ALT and LDH (Lactate dehydrogenase), all these result was further confirmed by histopathological studies of aorta which showed marked reduction in development of atherosclerosis plaque in the pre-treatment group. The result of the present study concluded that Centella asiatica has significant protection against STZ and HFD induced hyperlipidemia and diabetes which was compared to standard drugs i.e. atorvastatin and metformin.

\section{Bibliography}

1. Shaw JE., et al. "Global estimates of the prevalence of diabetes for 2010 and 2030". Diabetes Research and Clinical Practice 87 (2010): 4-14.

2. World Health Organization. Global Report on Diabetes. Geneva: World Health Organization (2016).

3. Mohsina FP., et al. "Evaluation of Anti diabetic activity of Ichnocarpus frutescens". International Journal in the field of Pharmacy, Biotechnology 4.2 (2018): 1-12.

4. Patel Rakesh., et al. "A Review on Role of Natural Phytoconstituents in the Management of Diabetes Mellitus" 51 (2021): 1231-1242.

5. http://www.metacure.com/about-diabetes-2/type-2-diabetes-mellitus-t2dm
6. Bertoli A., et al. "Lipid profile, BMI, body fat distribution and aerobic fitness in men with metabolic syndrome". Actadiabetologica 40.1 (2003): 130-133

7. Fazio S and Linton M F. "The role of fibrates in managing hyperlipidemia: mechanisms of action and clinical efficacy". Current Atherosclerosis Reports 6.2 (2004): 148-157.

8. Rao Priya Shaival and Rao K Shaival. "Review on Centellaasiatica: A Wonder Drug". International Journal of Pharmaceutical and Chemical Sciences 1.3 (2012): 1020-1026.

9. Leung AY and Foster S. "Encyclopedia of Common Naturallngredients Used in Food, Drugs, and Cosmetics". 2nd edition. John Wiley and Son, NY (1998): 284.

10. www.holosweet.com/unanai/efficacyunanai.htm

11. Ayurveda health benefits of mandukaparni (Centellaasiatica)gotu kola by Dr. savitasuri 10-Apr- (2013).

12. V Gayathri., et al. "Anti-diabetic activity of ethanol of Centellaasiatica (L.)Urban (whole plant) in streotozotocin-induced diabetic rats, isolation of an active fraction and toxicity evalution of extract". International Journal of Medicinal and Aromatic Plants 1.3 (2011): 278-286.

13. Lee S J., et al. "Hypolipidemic and hypoglycemic effects of OrostachysjaponicusA. Berger extracts in streptozotocin-induced diabetic rats". Nutrition 5.4 (2011): 301-307.

14. K Srinivasan., et al. "Combination of high-fat diet-fed and lowdose streptozotocin-treated rat: A model for type 2 diabetes and pharmacological screening". Pharmacological Research 52 (2005): 313-320.

15. C Vijaya., et al. "Lipid lowering activity of ethanolic extract of leaves of Aeglemarmelos (Linn.) in Hyperlipidaemic models of Wistar Albino Rats". Indian Journal of Experimental Biology 47 (2009): 182-185.

16. Maiti Rajkumar., et al. "Attenuation of Hyperglycemia and Hyperlipidemia in Streptozotocin- Induced Diabetic Rats by Aqueous Extract of Seed of Tamarindusindica". Biological and Pharmaceutical Bulletin 28.7 (2005): 1172-1176.

\section{Volume 5 Issue 9 September 2021} (C) All rights are reserved by Mohd Ayyub., et al. 